\title{
Traduire
}

Une eutre perspective sur $r$ tatadciction

Revue française de la traduction

$244 \mid 2021$

Des jeux et des mots

\section{Tradiludik : votre carrière est en jeu !}

Julien Ramina, Séverine George et Marie-Laure Faurite

\section{(2) OpenEdition \\ Journals}

Édition électronique

URL : https://journals.openedition.org/traduire/2343

DOI : 10.4000/traduire.2343

ISSN : 2272-9992

Éditeur

Société française des traducteurs

Édition imprimée

Date de publication : 15 juin 2021

Pagination : 87-93

ISSN : 0395-773X

\section{Référence électronique}

Julien Ramina, Séverine George et Marie-Laure Faurite, «Tradiludik : votre carrière est en jeu! », Traduire [En ligne], 244 | 2021, mis en ligne le 15 juin 2021, consulté le 01 juillet 2021. URL : http:// journals.openedition.org/traduire/2343 ; DOI : https://doi.org/10.4000/traduire.2343 


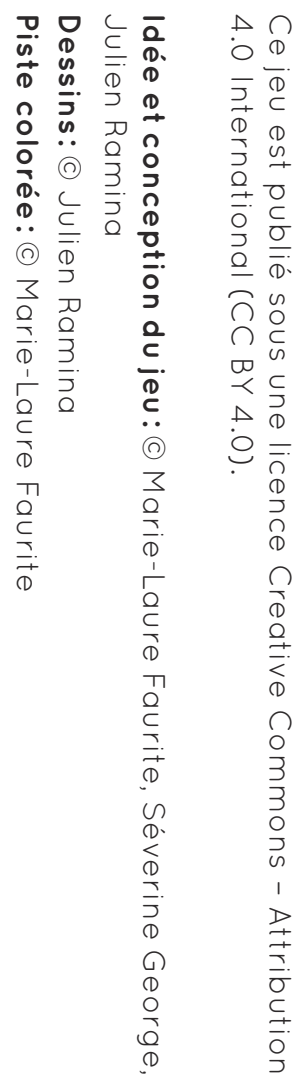

$\stackrel{\wedge}{\mathrm{Q}} \stackrel{0}{\subset}+3$

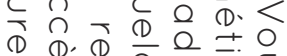
$N_{1} \backsim \leq 0 \subset$ $<\omega \subset \subset \cap T$ $O \subset$ (D) ज $\bigcirc \subset<<$ $\subset \supseteq \subset \supseteqq \top \mathrm{O}$ (1) U 万 D

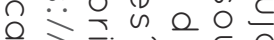

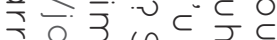
(D. $\subset$ (D) $\bigcirc$ D $\underline{D}$. T $\mathrm{I}$ 的 (D) $\vec{O}+\vec{D}+\mathbb{T}$

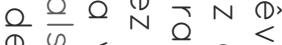
(D) $\backsim<\subset \stackrel{\varrho}{\circ} \stackrel{\circ}{\circ}$

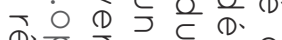
(D) $O$ जे

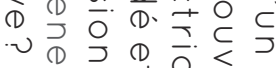

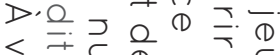

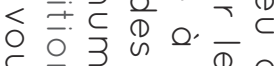

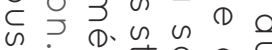

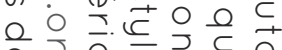
(1) $\widehat{0}$ 응

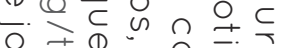

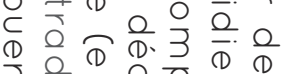

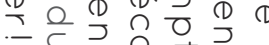
$\overline{\bar{N}}=\stackrel{\circ}{\bar{\sigma}} \overrightarrow{0}$ 잉

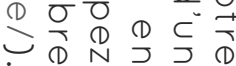
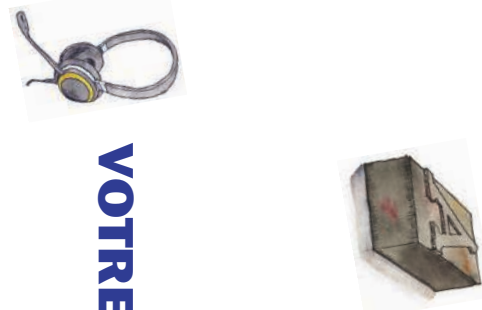

(1)

m

,

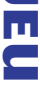

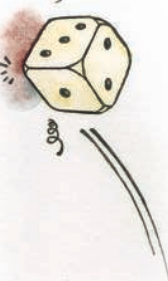




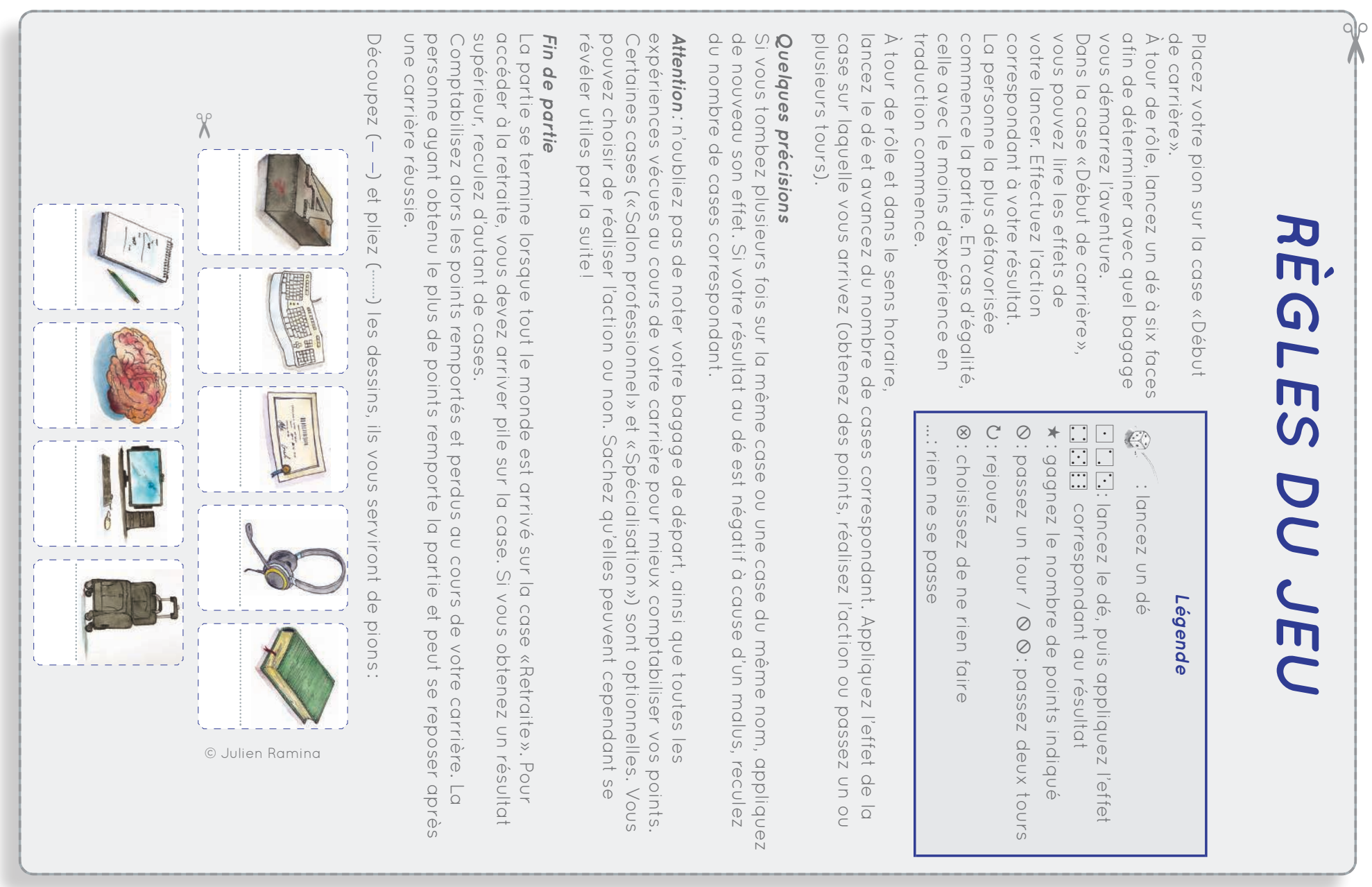



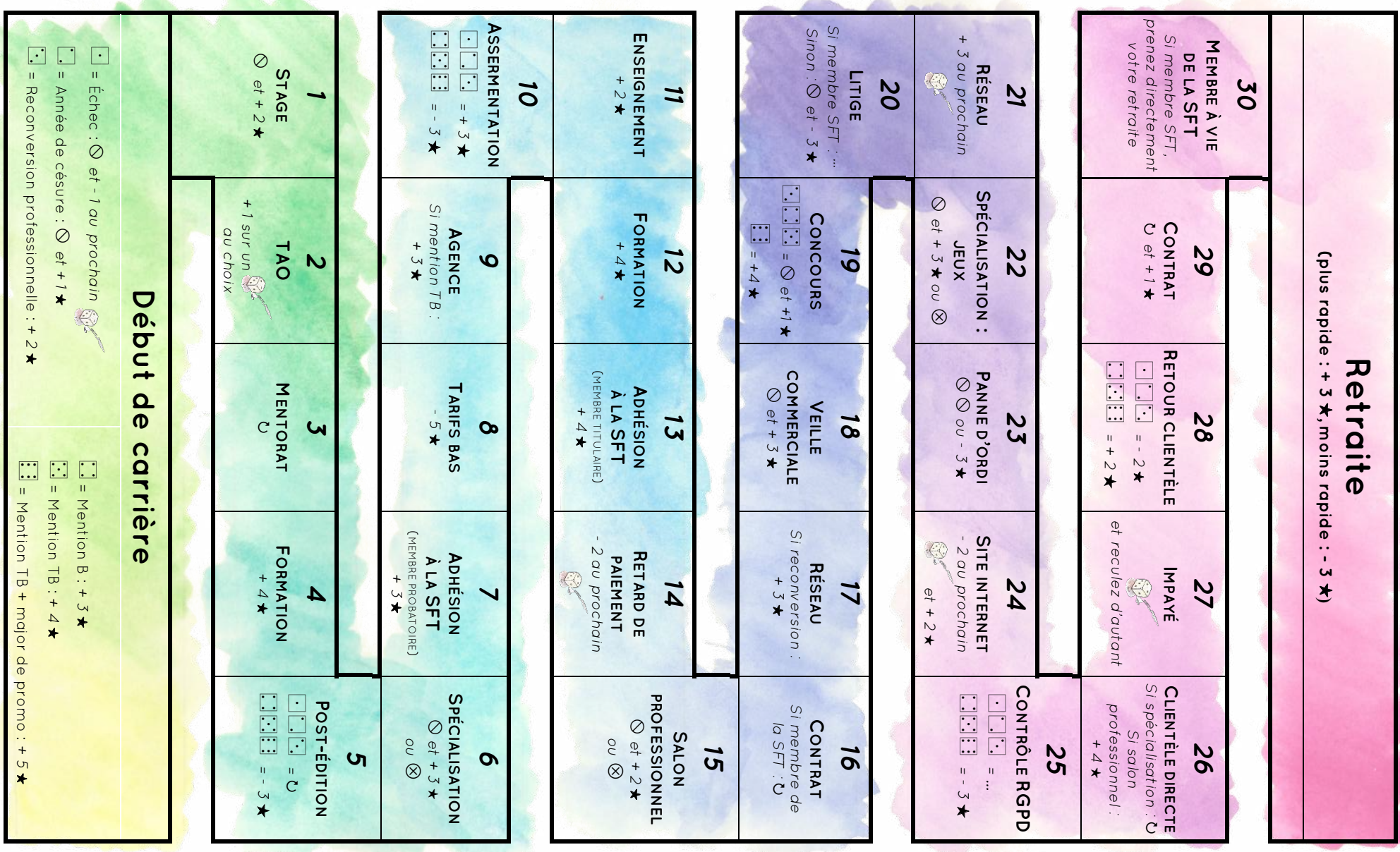


\begin{tabular}{|c|c|c|}
\hline$N^{\circ}$ & INTITULÉ & EXPLICATIONS \\
\hline & $\begin{array}{l}\text { DÉPART } \\
\quad= \\
\text { début de carrière }\end{array}$ & $\begin{array}{l}\text { De nombreux diplômes de traduction existent dans le monde. Assurez-vous de faire les bons choix et d'opter } \\
\text { pour une formation professionnalisante, reconnue et complète. } \\
\text { En fonction de votre résultat au dé, obtenez un avantage plus ou moins intéressant par rapport à vos collègues. }\end{array}$ \\
\hline 1 & Stage & $\begin{array}{l}\text { Un stage représente un atout certain que vous pouvez valoriser auprès des agences et autres } \\
\text { commanditaires. }\end{array}$ \\
\hline 2 & TAO & $\begin{array}{l}\text { Certes, la traduction assistée par ordinateur (TAO) représente un investissement, mais elle vous fait gagner } \\
\text { en productivité. } \\
\text { Ajoutez } 1 \text { au résultat d'un lancer une fois au cours de la partie. }\end{array}$ \\
\hline 3 & Mentorat & $\begin{array}{l}\text { Les programmes de mentorat, tels que le programme Boussole de la SFT, vous font bénéficier de l'aide et des } \\
\text { conseils avisés de collègues disposant de plus d'expérience. }\end{array}$ \\
\hline 4 & Formation & $\begin{array}{l}\text { Il est important de vous former tout au long de votre carrière pour suivre l'évolution du métier, proposer à } \\
\text { votre clientèle des services sans cesse renouvelés et être à l'aise avec les activités annexes. }\end{array}$ \\
\hline 5 & Post-édition & $\begin{array}{l}\text { La post-édition est maintenant courante en traduction. Une connaissance et une utilisation éclairée de la } \\
\text { traduction automatique vous permettent de tirer profit de ses avantages et d'entamer un dialogue avec vos } \\
\text { commanditaires. } \\
\text { Lancez le dé et appliquez le résultat correspondant. }\end{array}$ \\
\hline 6 & Spécialisation & $\begin{array}{l}\text { Une spécialisation dans un secteur précis, voire dans un domaine de niche, vous démarque de vos collègues } \\
\text { et est appréciée par votre clientèle. } \\
\text { Choisissez de vous spécialiser ou non. }\end{array}$ \\
\hline 7 & Adhésion à la SFT & $\begin{array}{l}\text { En tant que membre de la SFT, vous bénéficiez de services et d'un accompagnement privilégiés, d'un réseau } \\
\text { d'échanges et de formations a tarif préférentiel. Le statut de membre probatoire est réservé aux linguistes en } \\
\text { activité depuis moins d'un an. }\end{array}$ \\
\hline 8 & Tarifs bas & $\begin{array}{l}\text { Réussir à négocier des tarifs attractifs, même si cela peut être compliqué en début de carrière, est crucial } \\
\text { pour valoriser votre travail à sa juste valeur, défendre le métier et vivre de votre activité. }\end{array}$ \\
\hline 9 & Agence & $\begin{array}{l}\text { Une candidature soignée et complète peut faire la différence auprès d'une agence, et une mention obtenue } \\
\text { au diplôme est valorisée. }\end{array}$ \\
\hline
\end{tabular}




\begin{tabular}{|c|c|c|}
\hline 10 & Assermentation & $\begin{array}{l}\text { L'assermentation est accordée par une Cour de cassation ou des cours d'appel et vous permet d'assister } \\
\text { la justice et de fournir des traductions certifiées. La demande se fait sur dossier et peut être acceptée ou } \\
\text { refusée. } \\
\text { Lancez le dé et appliquez le résultat correspondant. }\end{array}$ \\
\hline 11 & Enseignement & $\begin{array}{l}\text { En parallèle de votre activité de traduction, vous pouvez partager votre passion et votre expérience auprès } \\
\text { de linguistes en formation. }\end{array}$ \\
\hline 12 & Formation & $\begin{array}{l}\text { La formation permet de suivre l'évolution du métier, de maîtriser de nouveaux outils et de valoriser votre } \\
\text { travail en mettant en avant vos compétences. En effet, l'enquête } 2009 \text { de la SFT notait la corrélation entre } \\
\text { formation et tarif: "Les collègues qui suivent le plus de formations ont des tarifs au mot plus élevés.» }\end{array}$ \\
\hline 13 & Adhésion à la SFT & $\begin{array}{l}\text { En tant que membre de la SFT, vous bénéficiez de services et d'un accompagnement privilégiés, d'un réseau } \\
\text { d'échanges et de formations à tarif préférentiel. }\end{array}$ \\
\hline 14 & $\begin{array}{l}\text { Retard de } \\
\text { paiement }\end{array}$ & $\begin{array}{l}\text { Les retards et problèmes de paiement peuvent avoir de lourdes répercussions sur votre activité et votre } \\
\text { trésorerie. Veillez à bien suivre vos projets terminés et à fournir des factures comportant toutes les mentions } \\
\text { légales. }\end{array}$ \\
\hline 15 & $\begin{array}{l}\text { Salon } \\
\text { professionnel }\end{array}$ & $\begin{array}{l}\text { Participer à des salons professionnels regroupant des spécialistes de votre domaine vous permet de } \\
\text { rencontrer des prospects, de vous tenir au courant de l'actualité et d'accroître votre visibilité. }\end{array}$ \\
\hline 16 & Contrat & $\begin{array}{l}\text { En tant que membre de la SFT, vous bénéficiez d'une visibilité accrue grâce à l'annuaire du site de la SFT, ce } \\
\text { qui vous permet de décrocher des contrats. }\end{array}$ \\
\hline 17 & Réseau & $\begin{array}{l}\text { Le réseau est très important pour trouver de nouveaux projets, recommander des collègues et sortir de } \\
\text { l'isolement. } \\
\text { Grâce à votre reconversion professionnelle, vous disposez d'un réseau d'ex-collègues et spécialistes très } \\
\text { enrichissant. }\end{array}$ \\
\hline 18 & Veille commerciale & $\begin{array}{l}\text { Une présence sur les réseaux sociaux et dans les salons professionnels augmente votre visibilité, élargit votre } \\
\text { carnet d'adresses et vous donne accès aux évolutions de votre secteur. }\end{array}$ \\
\hline 19 & Concours & $\begin{array}{l}\text { Certains postes sont uniquement accessibles sur concours. Soigner votre candidature et préparer les } \\
\text { épreuves nécessite du temps, mais permet de développer vos compétences. Attention: le taux de réussite est } \\
\text { limité! } \\
\text { Lancez le dé et appliquez le résultat correspondant. }\end{array}$ \\
\hline
\end{tabular}




\begin{tabular}{|c|c|c|}
\hline 20 & Litige & $\begin{array}{l}\text { En tant que membre de la SFT, vous pouvez contacter son service juridique qui vous conseille et vous } \\
\text { accompagne dans vos démarches en cas de non-paiement. }\end{array}$ \\
\hline 21 & Réseau & $\begin{array}{l}\text { Les rencontres au cours de formations, d'événements SFT ou de salons permettent de renforcer la cohésion } \\
\text { entre linguistes, mais également d'entretenir des relations professionnelles avec des commanditaires. }\end{array}$ \\
\hline 22 & $\begin{array}{l}\text { Spécialisation: } \\
\text { jeux }\end{array}$ & $\begin{array}{l}\text { La traduction touche même les domaines les plus inédits. Le secteur du jeu nécessite un travail dynamique et } \\
\text { créatif, mais implique un degré de technicité très élevé. } \\
\text { Choisissez de vous spécialiser ou non. }\end{array}$ \\
\hline 23 & Panne d'ordi & $\begin{array}{l}\text { Une panne d'ordinateur ou de matériel peut frapper à tout moment. Prévoyez une alternative et sauvegardez } \\
\text { régulièrement vos données. } \\
\text { Choisissez l'une des alternatives. }\end{array}$ \\
\hline 24 & Site internet & $\begin{array}{l}\text { Disposer d'un site internet moderne, bien référencé (grâce au SEO) et valorisant votre activité est gage de } \\
\text { professionnalisme et vous apporte de nouveaux commanditaires. }\end{array}$ \\
\hline 25 & Contrôle RGPD & $\begin{array}{l}\text { Le règlement général sur la protection des données codifie la collecte et le traitement des données } \\
\text { personnelles. Adoptez les bons réflexes. } \\
\text { Lancez le dé et appliquez le résultat correspondant. }\end{array}$ \\
\hline 26 & Clientèle directe & $\begin{array}{l}\text { Afin d'attirer une clientèle directe, n'hésitez pas à formuler une offre très spécialisée et à rencontrer vos } \\
\text { prospects sur des salons et autres événements professionnels. }\end{array}$ \\
\hline 27 & Impayé & $\begin{array}{l}\text { Votre carrière peut être ponctuée de problèmes d'impayés, pensez à vous protéger contre cette éventualité } \\
\text { et à demander des acomptes pour les projets conséquents. }\end{array}$ \\
\hline 28 & Retour clientèle & $\begin{array}{l}\text { Après la livraison d'un gros projet, vous attendez les retours des commanditaires. } \\
\text { Lancez le dé et appliquez le résultat correspondant. }\end{array}$ \\
\hline 29 & Contrat & $\begin{array}{l}\text { Les efforts que vous menez pour démarcher régulièrement et contacter de nouveaux prospects peuvent } \\
\text { déboucher sur un nouveau contrat. }\end{array}$ \\
\hline 30 & $\begin{array}{l}\text { Membre à vie de } \\
\text { la SFT }\end{array}$ & $\begin{array}{l}\text { Si vous avez adhéré à la SFT pendant toute votre carrière, vous pouvez devenir membre à vie. Ce statut est } \\
\text { réservé aux membres ayant cotisé pendant plus de } 35 \text { ans et ayant liquidé leur retraite. } \\
\text { Si vous êtes membre de la SFT, allez directement à la case «Retraite». }\end{array}$ \\
\hline & $\begin{array}{l}\text { ARRIVÉE } \\
\quad= \\
\text { retraite }\end{array}$ & $\begin{array}{l}\text { Après une carrière riche en rebondissements et en émotions, vous prenez enfin votre retraite. } \\
\text { La première personne qui arrive sur la case «Retraite» remporte } 3 \text { points. La dernière personne arrivée perd } \\
3 \text { points. }\end{array}$ \\
\hline
\end{tabular}



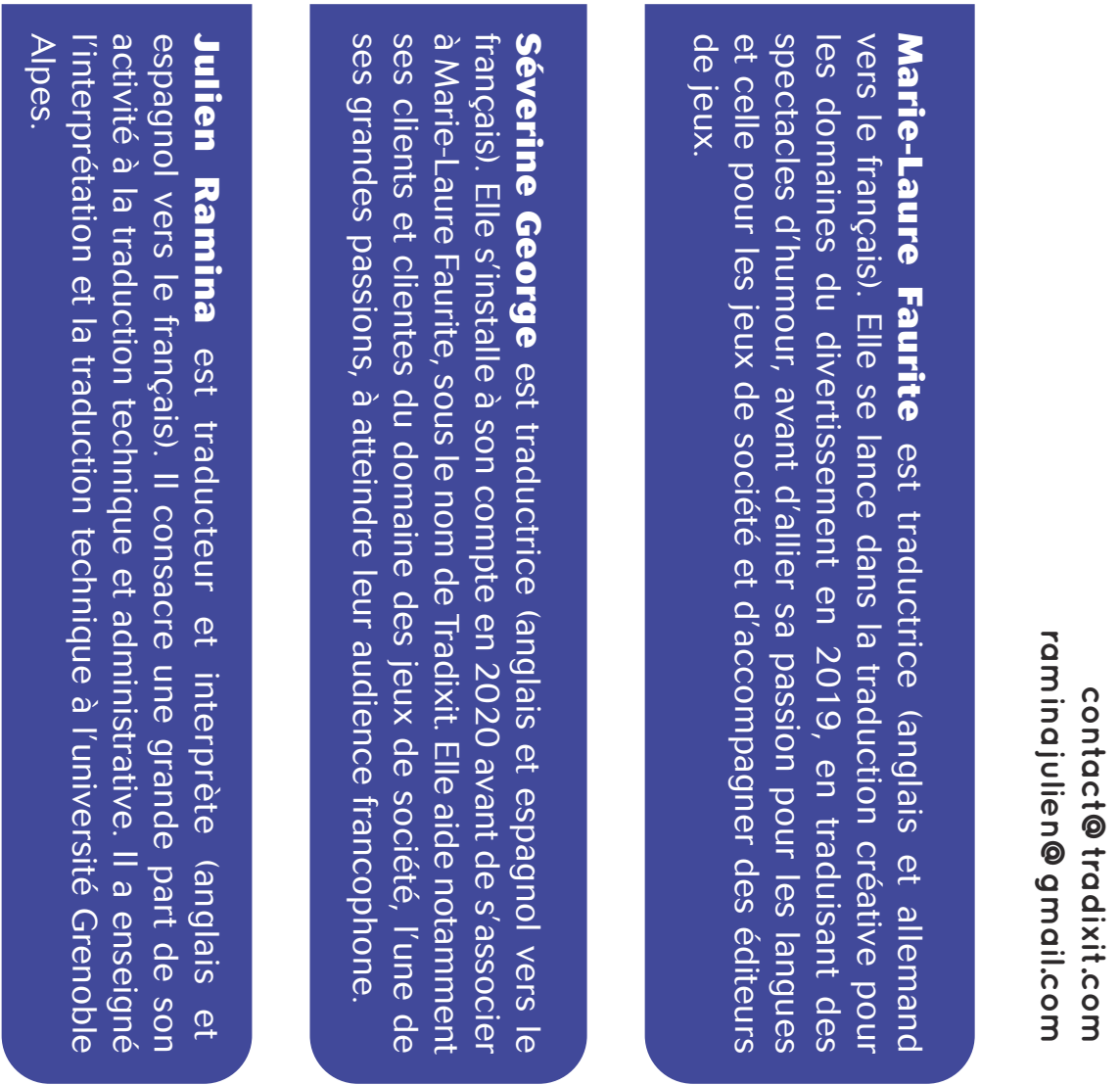